\title{
Diagnostic validity and triage concordance of a physiotherapist compared to physicians' diagnoses for common knee disorders
}

S. Décary ${ }^{1,2^{*}}$, M. Fallaha ${ }^{3}$, B. Pelletier ${ }^{3}$, P. Frémont ${ }^{4}$, J. Martel-Pelletier ${ }^{5}$, J.-P. Pelletier ${ }^{5}$, D. E. Feldman ${ }^{1}$, M.-P. Sylvestre ${ }^{6}$, P.-A. Vendittoli $i^{2,3}$ and F. Desmeules ${ }^{1,2}$

\begin{abstract}
Background: Emergence of more autonomous roles for physiotherapists warrants more evidence regarding their diagnostic capabilities. Therefore, we aimed to evaluate diagnostic and surgical triage concordance between a physiotherapist and expert physicians and to assess the diagnostic validity of the physiotherapist's musculoskeletal examination (ME) without imaging.

Methods: This is a prospective diagnostic study where 179 consecutive participants consulting for any knee complaint were independently diagnosed and triaged by two evaluators: a physiotherapist and one expert physician (orthopaedic surgeons or sport medicine physicians). The physiotherapist completed only a ME, while the physicians also had access to imaging to make their diagnosis. Raw agreement proportions and Cohen's kappa (k) were calculated to assess inter-rater agreement. Sensitivity (Se) and specificity (Sp), as well as positive and negative likelihood ratios (LR+/-) were calculated to assess the validity of the ME compared to the physicians' composite diagnosis.

Results: Primary knee diagnoses included anterior cruciate ligament injury $(n=8)$, meniscal injury $(n=36)$, patellofemoral pain $(n=45)$ and osteoarthritis $(n=79)$. Diagnostic inter-rater agreement between the physiotherapist and physicians was high ( $\mathrm{k}=0.89 ; 95 \% \mathrm{Cl}: 0.83-0.94)$. Inter-rater agreement for triage recommendations of surgical candidates was good ( $\mathrm{k}=0.73 ; 95 \% \mathrm{Cl}: 0.60-0.86$ ). Se and Sp of the physiotherapist's ME ranged from 82.0 to 100.0\% and 96.0 to $100.0 \%$ respectively and LR+/- ranged from 23.2 to 30.5 and from 0.03 to 0.09 respectively.

Conclusions: There was high diagnostic agreement and good triage concordance between the physiotherapist and physicians. The ME without imaging may be sufficient to diagnose or exclude common knee disorders for a large proportion of patients. Replication in a larger study will be required as well as further assessment of innovative multidisciplinary care trajectories to improve care of patients with common musculoskeletal disorders.
\end{abstract}

Keywords: Diagnosis, Knee disorders, Physiotherapist

\footnotetext{
* Correspondence: simon.decary.1@umontreal.ca; decary.simon@gmail.com

${ }^{1}$ School of Rehabilitation, Faculty of Medicine, University of Montreal, Montreal, QC, Canada

${ }^{2}$ Orthopaedic Clinical Research Unit, Maisonneuve-Rosemont Hospital

Research Center, Centre intégré universitaire de santé et de services sociaux

de l'Est-de-l'̂le-de-Montréal, Montreal, QC, Canada

Full list of author information is available at the end of the article
} 


\section{Background}

Knee disorders are a common reason for seeking diagnosis and management in primary care and can significantly impact quality of life of individuals [1-3]. However, evidence shows the limited ability of medical providers to perform an appropriate physical examination to make a diagnosis $[4,5]$. This has led to an overreliance on imaging or inappropriate referral to specialists to confirm a diagnosis, which incurs increasing health care costs and unnecessary delays to initiate conservative care [4-8]. Models of care in which physiotherapists act as first contact providers have been proposed [4, 9-11]. In these models, physiotherapists act as consultants who evaluate the patient, make a diagnosis and offer conservative care or refer to other providers $[4,9-11]$.

To adequately take on these autonomous roles, physiotherapists need to be able to provide a valid clinical diagnostic impression and be able to refer accurately patients to other providers or surgical candidates to orthopaedic surgeons; they need do this in manner that is as effective as physicians with expertise in musculoskeletal disorders would do [1]. Moore et al. demonstrated the equivalence between physiotherapists and orthopaedic surgeons for the clinical diagnosis of common musculoskeletal disorders [5]. When compared to magnetic resonance imaging (MRI) results, the diagnostic agreement of the physiotherapists was almost as high as the orthopaedic surgeons (raw agreement: $74.5 \%$ compared to $80.8 \%$ ), and it was superior to non-orthopaedic providers such as primary care physicians (35.4\%) [5]. A systematic review reported that, based on moderate quality studies, inter-rater agreement kappa values ranged from 0.69 to 1.00 for diagnostic agreement between physiotherapists and orthopaedic surgeons and kappa values ranging from 0.52 to 0.70 for the triage of surgical candidates, indicating moderate to high agreement between providers [12].

However, in many primary care settings, imaging may be difficult to obtain rapidly or physiotherapists may not be allowed to order imaging and must therefore rely exclusively on musculoskeletal examination (ME) when assessing patients. Jackson et al. concluded in a metaanalysis including 35 diagnostic studies, not specific to physiotherapists, that a complete ME demonstrates adequate validity to include or exclude common knee disorders when compared to imaging or arthroscopic findings $[1,13]$. However, it is not known whether this also applies to physiotherapists.

Therefore, the objectives of this study were to 1) evaluate agreement on the diagnosis and surgical triage between a physiotherapist using a standardized ME without the use of imaging results and physicians and 2) to assess the validity of the physiotherapist's ME to diagnose common knee disorders.

\section{Methods}

\section{Study design and settings}

This study is part of a larger multi-center prospective diagnostic cohort study that aims to identify the optimal combination of elements from the history and physical examination for the diagnosis of common knee disorders. Recruitment took place in an outpatient orthopaedic clinic and a primary care family medicine clinic. All consecutive patients consulting one of the participating physicians for a new knee complaint between November 2014 and January 2016 were recruited. Also, we included participants from a university community (students, teaching staff and other personnel) if they sought a diagnosis and care for a current knee complaint. These participants received an email invitation to participate from September 2015 to January 2016. The present study, its design, methodology and reporting of results is based on the Standards for Reporting Diagnostic Accuracy Studies 2015 (STARD) $[14,15]$. The study was approved by the hospital's ethics committee. The study was explained by the physiotherapist to all participants and written informed consent was obtained from them prior to consultation.

\section{Participants}

Inclusion criteria were: 18 years of age or older, consulting for a knee complaint for which they sought diagnosis, and being able to understand and speak French. Patients previously diagnosed and treated by one of the participating physicians were excluded to ensure that the patient did not reveal their previous diagnosis to the physiotherapist. We also excluded patients who had undergone lower limb surgery in the past six months, patients with a knee arthroplasty or who presented with more than two lower limb pathologies in addition to the one for which they were consulting or if they were diagnosed with any systemic inflammatory disorder.

\section{Data collection procedure Patients' characteristics and history elements}

All clinical settings had the same data collection procedure. Upon arrival at the clinic, participants answered a questionnaire which included age, sex and anthropometric data (weight and height) to allow calculation of body mass index (BMI), duration of symptoms, history of the lesion (traumatic or non-traumatic), and presence of bilateral knee pain. Participants also completed the Knee Injury and Osteoarthritis Outcome Score (KOOS), a validated 42-item self-report questionnaire that assesses pain, symptoms, function in daily living, function in sport and recreation and knee-related quality of life [16-18]. Psychological distress was assessed using the Kessler-6 screening scale for serious mental disorders $[19,20]$. 


\section{Physical examination}

Each participant was then independently assessed by two evaluators: a physiotherapist and one of the four physicians. The two evaluations were completed on the same day with a fifteen-minute interval between each. The physiotherapist always evaluated the participants prior to the physicians in a separate room. Both the physiotherapist and the physicians were blinded to each other results. Following the physiotherapist's ME, the patients' pain was evaluated using a three-point likert scale (light, moderate, severe) and they were withdrawn from the study if their pain was moderately or severely increased compared to the start of the evaluation. The physician then proceeded to his independent history taking and physical examination.

\section{Diagnosis, reference standard and triage options}

After independently seeing the patient, the physiotherapist and the physician each completed a separate form where they indicated their primary and, when applicable secondary diagnosis. The physiotherapist was blinded to imaging results and therefore determined his diagnosis on the sole basis of his ME [21].

As the reference standard, the physicians had access to imaging to establish their diagnosis and they performed their own analysis of the relevant imaging results. All participants were required to have a radiograph of their knee that included the following three views: weightbearing antero-posterior view with lateral and skyline views [22]. Magnetic resonance imaging (MRI) was required when the physician suspected a ligament injury, a meniscal injury or any other uncertain diagnoses. If participants already had recent radiographs within 3 months of their participation or MRI results within 6 months with suitable views or scans that allow adequate interpretation and grading by the physician, these results were used. If the physician doubted that the imaging did not reflect the current stage of pathology another test was ordered. The physician made a final primary and secondary (if necessary) composite diagnosis based on the patient's history, physical tests and imaging results [23]. This final composite diagnosis was considered the reference standard against which the physiotherapist's diagnoses were compared to all participants. We also compared the physiotherapist's diagnoses to the imaging diagnoses only as a secondary reference standard [24-27].

Lastly, both the physiotherapist and physicians independently selected the triage option - conservative, surgical or undecided - for the patients. For patients seen by the sports medicine physicians in the family medicine unit or from the university community, the patients were considered surgical cases if the physician considered that all other options would not be adequate and that requesting a surgical consultation was the proper conduct.

\section{Standardisation and evaluators' experience}

Before the start of the study, physicians met with the research personnel to familiarize with the study protocol and verify if their usual practice differed from the other evaluators to improve concordance. All practitioners participated in the standardization of the techniques, interpretation of the physical tests and definition of the related diagnoses and all agreed to comply with the proposed definitions during their respective evaluation. The physiotherapist had one year of clinical experience. The four participating physicians (two orthopaedic surgeons and two sports medicine physicians [1]) each had more than 20 years of experience in the diagnosis and management of knee disorders.

\section{Sample size}

We calculated the sample size to detect an overall interrater Kappa value for the overall diagnostic concordance greater than 0.80 assuming a two-tailed null hypothesis for a Kappa equal to 0.4 or less [28-30]. We estimated the proportions of positives agreement for knee disorders diagnoses and expected Kappa values based on a previous cohort from a similar setting recuited by our team [8]; the required sample size is set at 71 patients considering a $80 \%$ power [28-30].

\section{Statistical analysis}

We used descriptive statistics to present the participants' characteristics. All primary diagnoses were classified using five common categories: 1- ACL injury; 2- meniscal injury; 3- patellofemoral pain; 4- osteoarthritis; 5- others [31, 32]. In the event where the physiotherapist and the physician disagreed on the primary diagnosis, the secondary diagnoses were taken into account to further evaluate diagnostic concordance. To measure the inter-rater agreement for the diagnostic categories and triage recommendations between the physiotherapist and the physicians, proportions of raw agreement and Cohen's Kappas with associated 95\% confidence intervals (CI) were calculated. Because Kappa values may become biased due to high or low prevalence of concordant cases compared or non-concordant cases, bias index and prevalence index were calculated where 0 indicates no bias and 1 a high bias [31, 33]. Prevalence and Bias Adjusted Kappas (PABAK) were calculated for each diagnostic category to correct for these potential biases [31,33]. Interpretation of inter-rater agreement was made according to the Landis and Koch scale in which 0 indicates poor agreement, 0-0.2 slight, 0.2-0.4 fair, 0.4-0.6 moderate, $0.6-0.8$ substantial or good and $>0.8$ almost perfect or high agreement $[28,31]$. For the validity of the ME, we compared the final diagnosis proposed by the physiotherapists' ME with the physicians' composite final diagnosis (reference standard) based on the ME, radiographs 
and MRI results when needed. Sensitivity (Se), specificity (Sp) and likelihood ratios with 95\% CIs were calculated $[34,35]$. Se and Sp relate respectively to the proportion of true positives and true negatives when a test is performed [36, 37]. Positive and negative likelihood ratios were used to evaluate the diagnostic validity of the physiotherapist's physical examination compared to the physicians' composite diagnosis and the following cut-offs were used: 1-to include a disorder a $L R+\geq 5$ and 2- to exclude a disorder a LR- $\leq 0.2$ as they are reported to produce at least a moderate shift in post-test probability of having or not a certain disorder [37, 38]. Analysis was performed using SPSS version 21 (SPSS Inc., Chicago) and $\mathrm{R}$ version 3.2 .3 (packages epiR, irr and psych, http://cran.r-project.org/).

\section{Results}

Table 1 presents the characteristics of participants. Out of 198 eligible patients, five (2.5\%) refused to participate, 14 (7.1\%) were excluded before consultation and 179 (90.4\%) were included in the study (see Additional file 1: Appendix 1). None were excluded following the physiotherapist's evaluation because of increased pain or for

Table 1 Characteristics of participants $(n=179)$

\begin{tabular}{lll}
\hline Characteristics & $\mathrm{n}(\%)$ & mean (SD) \\
\hline Age (years) & & $49.9(16.1)$ \\
Sex & & \\
$\quad$ Female & $114(64)$ & \\
$\quad$ Male & $65(36)$ &
\end{tabular}

Body Mass Index $\left(\mathrm{Kg} \backslash \mathrm{m}^{2}\right)$

$29.1(6.5)$

Recruitment site

\begin{tabular}{|c|c|c|}
\hline Orthopaedic clinic & $142(80)$ & \\
\hline Family medicine unit & $15(8)$ & \\
\hline University community & $22(12)$ & \\
\hline History of trauma & $47(26)$ & \\
\hline Bilateral knee pain & $39(22)$ & \\
\hline \multicolumn{3}{|l|}{ Duration of pain at time of consultation } \\
\hline$<3$ months & $17(10)$ & \\
\hline 3-12 months & $45(25)$ & \\
\hline$\geq 12$ months & $117(65)$ & \\
\hline \multicolumn{3}{|c|}{ KOOS- Knee Injury and Osteoarthritis Outcome Score (\%) } \\
\hline Pain & & $58.6(19.7)$ \\
\hline Symptoms & & $71.0(19.6)$ \\
\hline Activity of Daily Living & & $66.1(21.8)$ \\
\hline Sports & & $31.4(24.8)$ \\
\hline Quality of Life & & $40.9(20.3)$ \\
\hline K6 psychological distress scale (/30) & & $26.0(4.5)$ \\
\hline
\end{tabular}

SD standard deviation; KOOS: 0 indicates a severe condition and 100 indicates a normal knee; K6: 6 indicates serious mental illness and 30 indicates no mental illness any reasons. Mean age was $49.9 \pm 16.1$ years old and most participants were female (63.7\%) with a mean BMI of $29.1 \pm 6.5 \mathrm{~kg} / \mathrm{m}^{2}$. The majority of participants were recruited from the orthopaedic clinic (79.3\%) and consulted for a non-traumatic disorder (73.7\%). Most participants had pain for over 3 months at the time of consultation (90.5\%). KOOS Sports and Quality of life domains were most severely affected $(31.4 \pm 24.8$ and 40.9 \pm 20.3$)$.

Primary clinical diagnoses made by the participating physicians (using ME and imaging) included: anterior cruciate ligament injury (ACL) $(n=8)$, meniscal injury $(n=36)$, patellofemoral pain (PFP) $(n=45)$, osteoarthritis (OA) $(n=79)$ and other diagnoses $(n=11)$ (Table 2). All participants $(n=179)$ had radiograph results and 70 participants had an MRI scan. Based on imaging results only,

Table 2 Clinical and imaging diagnoses of participants $(n=179)$

\begin{tabular}{|c|c|}
\hline & n (\%) \\
\hline \multicolumn{2}{|l|}{ Primary clinical composite diagnoses } \\
\hline Anterior cruciate ligament injury & $8(5)$ \\
\hline Meniscal injury & $36(20)$ \\
\hline Patellofemoral Pain Syndrome & $45(25)$ \\
\hline Osteoarthritis & $79(44)$ \\
\hline Other knee diagnoses & $11(6)$ \\
\hline \multicolumn{2}{|l|}{ Imaging findings and diagnoses } \\
\hline Osteoarthritis & $96(56)$ \\
\hline K-L Grade 1 & $14(15)$ \\
\hline K-L Grade 2 & $36(38)$ \\
\hline K-L Grade 3 & $19(20)$ \\
\hline K-L Grade 4 & $13(14)$ \\
\hline Meniscal tears (n) & $54(32)$ \\
\hline Medial meniscus & $49(91)$ \\
\hline Lateral meniscus & $8(15)$ \\
\hline Anterior cruciate ligament tears & $16(9)$ \\
\hline Complete & $6(37)$ \\
\hline Partial & $8(50)$ \\
\hline Unclear & $2(13)$ \\
\hline Posterior cruciate ligament tear & $1(1)$ \\
\hline Soleus tear & $1(1)$ \\
\hline Hamstring tendinopathy & $2(1)$ \\
\hline Medial collateral ligament tear & $1(1)$ \\
\hline
\end{tabular}

SD standard deviation; Clinical diagnoses are composite diagnoses made by physicians using both musculoskeletal examination and imaging; Others knee diagnoses included: contusion of the tibial plateau $(n=2), \mathrm{PCL}$ tear $(n=1)$, soleus tear $(n=1)$, psychosomatic origin $(n=1)$, muscular spasms linked to multiple sclerosis $(n=1)$, hamstring tendinopathy $(n=3)$, medial collateral ligament injury $(n=1)$, functional instability without meniscal or ACL injury $(n=1)$; Imaging diagnoses are based on imaging studies using radiograph or magnetic resonance imaging; Grades are for Kellgren-Lawrence scale in the most affected compartment; Radiographic OA was defined as K-L $\geq 1$ 
diagnoses included: OA $(n=96)$, meniscal tears $(n=54)$, ACL tears $(n=16)$ or others $(n=5)$.

Table 3 presents the concordance between the diagnosis made by the physiotherapist using only the ME and the composite diagnosis made by physicians using both $\mathrm{ME}$ and imaging or with the imaging diagnoses only. The overall raw agreement between the physiotherapist and the physicians' diagnosis was $92.2 \%$ with an high inter-rater agreement ( $\mathrm{k}=0.89,95 \% \mathrm{CI}$ : $0.83-0.94)$. Interrater agreement for specific knee disorders ranged from $\mathrm{K}=0.88$ to 0.94 . ACL injury and other diagnoses had fewer cases $(8 / 179$ and $11 / 179)$ which translated into a high prevalence index ( 0.91 and 0.89 , respectively). However, all PABAK estimates were included in the Cohen's kappa 95\% confidence intervals and were therefore not significantly different, which indicates that even when bias were present (i.e: prevalence of ACL injuries), this did not influence the Kappa estimate [31]. When comparing the physiotherapists' diagnosis with imaging only, raw agreement was slightly lower at $84.4 \%$ and interrater agreement was good ( $\kappa=0.77 ; 95 \%$ CI: $0.68-0.85)$.

Table 4 presents the diagnostic validity of the physiotherapist's standardized ME compared to the reference standard (physician's composite diagnosis) to discriminate between each knee disorders. Sensitivity ranged from 82 to $100 \%$ and was lowest for Others knee disorders. Specificity ranged from 96 to $100 \%$. Positive likelihood ratio ranged from 23.2 to 267.6 and all 95\% CI lower bounds were above 10.0. Negative likelihood ratio ranged from 0.00 to 0.18 and all $95 \%$ CI upper bounds were below $\mathrm{LR}-\leq 0.20$, except for PFP $(\mathrm{LR}-=0.23)$ and Others (LR- $=0.65)$. This indicates that the standardized ME moderately to highly increases post-test probability to diagnose or exclude common knee disorders.

Table 5 presents the concordance between the physiotherapist and the physician for the triage recommendation following consultation. Only 23 participants were considered as surgical candidates and six participants as uncertain by the physicians (see Additional file 2: Appendix 2). Of the 23 patients considered surgical candidates, twenty were evaluated by the orthopaedic surgeons and three by the sports medicine physicians. Among those deemed surgical candidates, five had an ACL tear, seven a meniscal tear and eleven had an OA diagnosis. The overall agreement between the physiotherapist and all physicians was 91.6\% with an inter-rater kappa of 0.73 (95\% CI: 0.60-0.86). Raw agreement for surgical cases was $91.3 \%$ with only two of 23 surgical cases misclassified by the physiotherapist as conservative (see Additional file 2: Appendix 2). Raw agreement for conservative care was $92.6 \%$ with 11 of 150 conservative care cases misclassified by the physiotherapist as surgical cases (see Additional file 2: Appendix 2).

\section{Discussion}

The objectives of our study were to evaluate the diagnostic and surgical triage agreement between a physiotherapist and physicians to assess the validity of the physiotherapist's musculoskeletal examination (ME) without the use of medical imaging to diagnose common knee disorders. We found high diagnostic agreement and good triage agreement as well as high diagnostic validity for the ME performed by the physiotherapist in patients suffering from common knee disorders and consulting in primary and secondary care settings.

Our results compare well with two previous studies in orthopaedic settings where high inter-rater diagnostic agreement between a physiotherapist and orthopaedic surgeons for the diagnosis of common knee disorders were reported ( $=0.80$ and $\mathrm{\kappa}=0.87$ (95\% CI: $0.79-0.94)$ ) $[8,32]$. Of note, in both these studies, the physiotherapist also had access to imaging results to support his ME which was not the case in our study [8,32]. Only fourteen patients $(n=14)$ out of $179(7.8 \%)$ were discordant between the physiotherapist using ME compared with the physicians' composite diagnosis. Discordant patients included one meniscus injury, four patellofemoral pain, seven osteoarthritis one patellar tendinopathy and one functional instability without meniscal or ACL injuries (see Additional file 3: Appendix 3). A possible cause includes a potentially more complex presentation (history

Table 3 Concordance between the physiotherapist and physicians' composite or imaging only diagnoses $(n=179)$

\begin{tabular}{|c|c|c|c|c|c|c|}
\hline & Raw agreement & Cohen's kappa & $95 \% \mathrm{Cl}$ & Bias Index & Prevalence Index & PABAK \\
\hline Overall concordance with the physicians' composite diagnoses & $92.2 \%(165 / 179)$ & 0.89 & $0.83-0.94$ & - & - & - \\
\hline$A C L$ injury & $100.0 \%(8 / 8)$ & 0.94 & $0.82-1.00$ & 0.01 & 0.91 & 0.99 \\
\hline Meniscal injury & $97.2 \%(35 / 36)$ & 0.88 & $0.80-0.93$ & 0.03 & 0.57 & 0.92 \\
\hline Patellofemoral pain & $91.1 \%(41 / 45)$ & 0.88 & $0.80-0.96$ & 0.05 & 0.50 & 0.91 \\
\hline Osteoarthritis & $91.1 \%(72 / 79)$ & 0.89 & $0.82-0.95$ & 0.02 & 0.14 & 0.89 \\
\hline Other knee disorders & $81.8 \%(9 / 11)$ & 0.89 & $0.75-1.00$ & 0.01 & 0.89 & 0.98 \\
\hline Overall concordance with imaging only diagnoses & $84.4 \%(151 / 179)$ & 0.77 & $0.68-0.85$ & - & - & - \\
\hline
\end{tabular}

$A C L$ anterior cruciate ligament. $95 \% \mathrm{Cl}$ : $95 \%$ confidence interval. PABAK is only calculated only for $2 \times 2$ tables. Others knee diagnoses included: contusion of the tibial plateau $(n=2)$, PCL tear $(n=1)$, soleus tear $(n=1)$, psychosomatic origin $(n=1)$, muscular spasms linked to multiple sclerosis $(n=1)$, hamstring tendinopathy $(n=3)$, medial collateral ligament injury $(n=1)$, functional instability in the absence of $\mathrm{ACL}$ or meniscal injury $(n=1)$ 
Table 4 Diagnostic validity of the musculoskeletal examination performed by the physiotherapist compared to the physicians' composite diagnosis

\begin{tabular}{lllll}
\hline & $\begin{array}{l}\text { Sensitivity } \\
(95 \% \mathrm{Cl})\end{array}$ & $\begin{array}{l}\text { Specificity } \\
(95 \% \mathrm{Cl})\end{array}$ & $\begin{array}{l}\text { Positive Likelihood Ratio } \\
(95 \% \mathrm{Cl})\end{array}$ & $\begin{array}{l}\text { Negative Likelihood Ratio } \\
(95 \% \mathrm{Cl})\end{array}$ \\
\hline $\begin{array}{l}\text { ACL injuries } \\
(n=8)\end{array}$ & $100.0 \%$ & $99.0 \%$ & 171.0 & 0.00 \\
Meniscal injuries & $(52.0-100.0)$ & $(97.0-100.0)$ & $(24.2-1207.0)$ & $(0.00-0.00)$ \\
$(n=36)$ & $97.0 \%$ & $96.0 \%$ & 23.2 & 0.03 \\
Patellofemoral pain & $(85.0-100.0)$ & $(91.0-98.0)$ & $(10.6-50.8)$ & $(0.00-0.20)$ \\
$(n=45)$ & $91.0 \%$ & $97.0 \%$ & 30.5 & 0.09 \\
Osteoarthritis & $(79.0-98.0)$ & $(93.0-99.0)$ & $(11.6-80.5)$ & $(0.04-0.23)$ \\
$(n=79)$ & $91.0 \%$ & $97.0 \%$ & 30.4 & 0.09 \\
$\begin{array}{l}\text { Others knee disorders } \\
(n=11)\end{array}$ & $(83.0-96.0)$ & $(91.0-99.0)$ & $(10.0-92.8)$ & $(0.04-0.19)$ \\
\hline
\end{tabular}

$A C L$ anterior cruciate ligament. 95\% Cl: 95\% confidence interval

and physical examination). Also, because our composite reference standard is done by only one expert, it is possible that the physiotherapist may not be the source of discordance. When comparing the physiotherapist's diagnosis to imaging diagnoses only, the agreement was somewhat lower supporting the notion that imaging results need to be corroborated with clinical findings from the ME and that these findings may be more important to make a diagnosis [32].

Another objective of our study was to evaluate the diagnostic validity of a ME performed without imaging support. This objective is important in the context where the need to rely on imaging may delay care either by the physiotherapist or a physician or the ordering of a given imaging may be altogether unnecessary to make a diagnosis and initiate appropriate care. Our results show that a ME performed by a physiotherapist without imaging could reach moderate to high diagnostic validity to diagnose or exclude common knee disorders and these results are comparable to already published evidence. Jackson et al. reported in their meta-analysis LR $+\geq 10$ for lateral meniscus, ACL injuries and cartilage lesions and $L R-\leq 0.20$ for lateral and medial meniscus and $\mathrm{LR}_{-}=0.27$ for $\mathrm{ACL}$ injury confirming the validity of a complete clinical examination performed by orthopaedic surgeons or sports medicine physicians [1]. What remains to be established is what is the optimal combination of history questions and physical examination tests results in

Table 5 Concordance between the physiotherapist and physicians for the triage recommendation following consultation ( $n=179$ )

\begin{tabular}{llll}
\hline & Raw agreement & Cohen's kappa & $95 \% \mathrm{Cl}$ \\
\hline Overall & $91.6 \%(164 / 179)$ & 0.73 & $0.60-0.86$ \\
$\begin{array}{l}\text { Surgical candidates } \\
\text { Conservative care }\end{array}$ & $91.3 \%(21 / 23)$ & & \\
candidates & $92.6 \%(139 / 150)$ & & \\
Uncertain & $66.7 \%(4 / 6)$ & & \\
\hline
\end{tabular}

95\% Cl: 95\% confidence interval the ME that is helpful to support the differential diagnosis of common knee disorders [39-43]. Nonetheless, the use of imaging may be warranted in more complex cases or to diagnose or exclude uncommon disorders when the expected recovery after initiation of care is not as predicted. In this situation, it is interesting to see that published evidence support physiotherapists to refer patients autonomously and appropriately to imaging $[5,8]$.

Our findings regarding concordance for triage recommendations of surgical candidates are consistent with three previous studies demonstrating good triage agreement with an orthopaedic surgeon (raw agreement: $87 \%$ and $91.8 \%$; $\mathrm{k}=0.77$; 95\% CI: $0.65-0.88$, respectively) $[8,32,44]$. As stated above, the physiotherapist had access only to the ME without imaging to make the triage recommendation. Recent evidence proposes that pain, functional limitations and clinical symptoms should be used as surgical eligibility criteria for ACL injuries [45], meniscal injuries [46, 47] and knee OA [48, 49] and not systematically rely on imaging results. In our study, $82 \%(116 / 142)$ of secondary care participants and $92 \%$ $(34 / 37)$ of primary care participants were referred to conservative care after their first consultation. Almost all of these patients (92.6\%) would have been appropriately triaged to conservative care directly by the physiotherapist based only on the ME, making their care trajectory likely more efficient. Interestingly, this suggests that our cohort spectrum and representativeness is balanced between primary care and a pure secondary or tertiary surgical setting. Therefore, a well-executed ME may provide appropriate findings to guide patients to the appropriate care and these results suggests the role of physiotherapists as qualified musculoskeletal experts for knee disorders $[44,50]$.

\section{Strengths and limitations}

Our prospective cohort was recruited from three different settings, both in primary and secondary care, allowing for a broad variety of patients with various knee disorders, 
thus limiting spectrum bias commonly encountered in other diagnostic studies [51]. However, most patients were recruited in orthopedic clinics and this may limit the applicability of the findings to other settings. Evaluators met prior to the initiation of the study to standardize techniques and interpretation of the physical tests and related diagnoses, but no formal evaluation of their skills was undertaken. Also, the physiotherapist always evaluated the patients prior to the physician and this may have increased the diagnostic concordance by sensitizing the patients even though evaluators remained blinded to each others results. Our composite reference standard included both musculoskeletal examination and imaging interpretation by experienced medical experts, which is considered clinically relevant in the study of musculoskeletal disorders [24-27]. However, only one physiotherapist and one of four medical experts evaluated each given participant and this limits the generalizability of our results. It must be noted that the physiotherapist in our study had only one year of clinical experience, which is suggestive of the appropriateness of physiotherapy training programs to adequately train therapists in musculoskeletal examination, but will require confirmation with more physiotherapists of diverse level of experience.

\section{Conclusions}

High diagnostic agreement and good triage of surgical candidates agreement was found between the physiotherapist and experienced physicians for various knee problems. Musculoskeletal examination without imaging performed by trained musculoskeletal providers may yield high diagnostic validity to discriminate between common knee disorders. This suggests the potential role of healthcare professionals such as physiotherapists in the development of multidisciplinary evaluation and triage strategies in the context of innovative and potentially more efficient care trajectories for patients with common musculoskeletal disorders. These results will require confirmation with a larger study, in other primary care settings, with a greater number of physiotherapists and for other musculoskeletal disorders.

\section{Additional files}

Additional file 1: Appendix 1. Flowchart of patients recruitment (DOCX $59 \mathrm{~kb}$ )

Additional file 2: Appendix 2. $3 \times 3$ table for triage of surgical candidates and conservative care. (DOCX $43 \mathrm{~kb}$ )

Additional file 3: Appendix 3. $2 \times 2$ tables for diagnoses of knee disorders. (DOCX $83 \mathrm{~kb}$ )

\section{Abbreviations}

ACL: Anterior cruciate ligament; LR: Likelihood ratio; NPV: Negative predictive value; OA: Osteoarthritis; PFP: Patellofemoral pain; PPV: Positive predictive value; Se: Sensitivity; Sp: Specificity

\section{Acknowledgements}

Not applicable.

\section{Funding}

This project has received funding from the Canadian Institute of Health Research (CIHR). SD obtained doctoral training award from Fonds de Recherche en Santé du Québec (FRQS). FD is a CIHR scholar.

\section{Availability of data and materials}

All $2 \times 2$ contingency table to replicate findings are provided in the manuscript's appendix. Databases can be obtained by contacting the corresponding author

\section{Authors' contributions}

SD, FD and PAV designed the study, analyzed the data and drafted the manuscript. PF, BP, MF supported the design of the musculoskeletal examination standardized procedures and diagnosed participants. JMP, JPP, DF, MPS supported the design of the study, appropriateness of data analysis and presentation of results. All authors read and approved the final manuscript.

\section{Ethics approval and consent to participate}

The study was approved by Hopital Maisonneuve-Rosemont ethics committee. The study was explained to all participants and written informed consent was obtained from them prior to consultation.

\section{Consent for publication}

Not applicable.

\section{Competing interests}

The authors declare that they have no competing interests.

\section{Publisher's Note}

Springer Nature remains neutral with regard to jurisdictional claims in published maps and institutional affiliations.

\section{Author details}

${ }^{1}$ School of Rehabilitation, Faculty of Medicine, University of Montreal, Montreal, QC, Canada. ${ }^{2}$ Orthopaedic Clinical Research Unit, Maisonneuve-Rosemont Hospital Research Center, Centre intégré universitaire de santé et de services sociaux de l'Est-de-l'Île-de-Montréal, Montreal, QC, Canada. ${ }^{3}$ Department of Surgery, Maisonneuve-Rosemont Hospital, University of Montreal, Montreal, QC, Canada. Centre intégré universitaire de santé et de services sociaux de l'Est-de-l'̂le-de-Montréal, Montreal, QC, Canada. ${ }^{4}$ Department of Rehabilitation, Faculty of Medicine, Laval University, Quebec City, QC, Canada. ${ }^{5}$ Osteoarthritis Research Unit, University of Montreal Hospital Research Center (CRCHUM), Montreal, QC, Canada. ${ }^{6}$ Department of Social Preventive Medicine, School of Public Health, Université de Montréal, Montreal, QC, Canada.

Received: 26 April 2017 Accepted: 1 November 2017

Published online: 14 November 2017

\section{References}

1. Jackson $J \mathrm{~L}, \mathrm{O}$ 'Malley $\mathrm{PG}$, Kroenke K. Evaluation of acute knee pain in primary care. Ann Intern Med. 2003;139(7):575-88.

2. Baker $P$, et al. Knee disorders in the general population and their relation to occupation. Occup Environ Med. 2003;60(10):794-7.

3. van Middelkoop M, et al. Knee complaints seen in general practice: active sport participants versus non-sport participants. BMC Musculoskelet Disord. 2008;9:36

4. Lau BH, et al. Utilization and cost of a new model of care for managing acute knee injuries: the Calgary acute knee injury clinic. BMC Health Serv Res. 2012;12:445.

5. Moore $\mathrm{JH}$, et al. Clinical diagnostic accuracy and magnetic resonance imaging of patients referred by physical therapists, orthopaedic surgeons, and nonorthopaedic providers. J Orthop Sports Phys Ther. 2005;35(2):67-71.

6. Swart NM, et al. Efficacy of MRI in primary care for patients with knee complaints due to trauma: protocol of a randomised controlled non-inferiority trial (TACKLE trial). BMC Musculoskelet Disord. 2014;15:63. 
7. Mather RC 3rd, et al. Cost-effectiveness analysis of the diagnosis of meniscus tears. Am J Sports Med. 2015;43(1):128-37.

8. Desmeules $F$, et al. Validation of an advanced practice physiotherapy model of care in an orthopaedic outpatient clinic. BMC Musculoskelet Disord. 2013;14:162.

9. Daker-White $G$, et al. A randomised controlled trial. Shifting boundaries of doctors and physiotherapists in orthopaedic outpatient departments. J Epidemiol Community Health. 1999;53(10):643-50.

10. de Gruchy A, Granger C, Gorelik A. Physical therapists as primary practitioners in the emergency department: six-month prospective practice analysis. Phys Ther. 2015;95(9):1207-16.

11. Bornhoft L, Larsson ME, Thorn J. Physiotherapy in primary care triage - the effects on utilization of medical services at primary health care clinics by patients and sub-groups of patients with musculoskeletal disorders: a case-control study. Physiother Theory Pract. 2015;31(1):45-52.

12. Desmeules F, et al. Advanced practice physiotherapy in patients with musculoskeletal disorders: a systematic review. BMC Musculoskelet Disord. 2012;13(1):107.

13. Décary $\mathrm{S}$, et al. Diagnostic validity of physical examination tests for common knee disorders: an overview of systematic reviews and metaanalysis. Phys Ther Sport. 2017;23:143-55.

14. Bossuyt PM, et al. STARD 2015: an updated list of essential items for reporting diagnostic accuracy studies. BMJ. 2015;351:h5527.

15. Simel DL, Rennie D, Bossuyt PM. The STARD statement for reporting diagnostic accuracy studies: application to the history and physical examination. J Gen Intern Med. 2008;23(6):768-74.

16. Collins NJ, et al. Measures of knee function: international knee documentation committee (IKDC) subjective knee evaluation form, knee injury and osteoarthritis outcome score (KOOS), knee injury and osteoarthritis outcome score physical function short form (KOOS-PS), knee outcome survey activities of daily living scale (KOS-ADL), Lysholm knee scoring scale, Oxford knee score (OKS), western Ontario and McMaster universities osteoarthritis index (WOMAC), activity rating scale (ARS), and Tegner activity score (TAS). Arthritis Care Res (Hoboken). 2011:63(Suppl 11):S208-28.

17. Esculier JF, Roy JS, Bouyer LJ. Psychometric evidence of self-reported questionnaires for patellofemoral pain syndrome: a systematic review. Disabil Rehabil. 2013;35(26):2181-90.

18. Peer MA, Lane J. The knee injury and osteoarthritis outcome score (KOOS): a review of its psychometric properties in people undergoing total knee arthroplasty. J Orthop Sports Phys Ther. 2013;43(1):20-8.

19. Kessler RC, et al. Screening for serious mental illness in the general population. Arch Gen Psychiatry. 2003;60(2):184-9.

20. Kessler RC, et al. Screening for serious mental illness in the general population with the $\mathrm{K} 6$ screening scale: results from the $\mathrm{WHO}$ world mental health $(\mathrm{WMH})$ survey initiative. Int J Methods Psychiatr Res. 2010;19(Suppl 1):4-22.

21. Whiting $P$, et al. The development of QUADAS: a tool for the quality assessment of studies of diagnostic accuracy included in systematic reviews. BMC Med Res Methodol. 2003;3:25.

22. Culvenor AG, et al. Patellofemoral osteoarthritis is prevalent and associated with worse symptoms and function after hamstring tendon autograft $\mathrm{ACL}$ reconstruction. Br J Sports Med. 2014:48(6):435-9.

23. Naaktgeboren CA, et al. Value of composite reference standards in diagnostic research. BMJ. 2013;347:f5605.

24. Reitsma JB, et al. A review of solutions for diagnostic accuracy studies with an imperfect or missing reference standard. J Clin Epidemiol. 2009;62(8):797-806.

25. Henschke $\mathrm{N}$, et al. The methodological quality of diagnostic test accuracy studies for musculoskeletal conditions can be improved. J Clin Epidemiol. 2014;67(4):416-24.

26. Barton CJ, et al. Kinematic gait characteristics associated with patellofemoral pain syndrome: a systematic review. Gait Posture. 2009;30(4):405-16.

27. Rutjes AW, et al. Evaluation of diagnostic tests when there is no gold standard. A review of methods. Health Technol Assess. 2007;11(50):iii. ix-51

28. Sim J, Wright CC. The kappa statistic in reliability studies: use, interpretation, and sample size requirements. Phys Ther. 2005;85(3):257-68.

29. Flack VF, Afifi AA, Lachenbruch PA, Schouten HJA. Sample size determinations for the two rater kappa statistic. Psychometrika. 1988:53:321-5.

30. Cantor AB. Sample-size calculation for Cohen's kappa. Psychol Methods. 1996:1:150-3.

31. Razmjou H, et al. Evaluation of an advanced-practice physical therapist in a specialty shoulder clinic: diagnostic agreement and effect on wait times. Physiother Can. 2013;65(1):46-55.
32. Aiken AB, McColl MA. Diagnostic and treatment concordance between a physiotherapist and an orthopedic surgeon-a pilot study. J Interprof Care. 2008;22(3):253-61.

33. Lange $T$, et al. The reliability of physical examination tests for the diagnosis of anterior cruciate ligament rupture-a systematic review. Man Ther. 2015; 20(3):402-11.

34. Simel DL, Samsa GP, Matchar DB. Likelihood ratios with confidence: sample size estimation for diagnostic test studies. J Clin Epidemiol. 1991:44(8):763-70.

35. Lubetzky-Vilnai A, Ciol M, McCoy SW. Statistical analysis of clinical prediction rules for rehabilitation interventions: current state of the literature. Arch Phys Med Rehabil. 2014;95(1):188-96.

36. Hing W, et al. Validity of the McMurray's test and modified versions of the test: a systematic literature review. J Man Manip Ther. 2009;17(1):22-35.

37. Jaeschke R, Guyatt GH, Sackett DL. Users' guides to the medical literature. III. How to use an article about a diagnostic test. B. What are the results and will they help me in caring for my patients? The evidence-based medicine working group. JAMA. 1994:271(9):703-7.

38. Hegedus EJ, Stern B. Beyond SpPIN and SnNOUT: considerations with dichotomous tests during assessment of diagnostic accuracy. J Man Manip Ther. 2009;17(1):E1-5.

39. Cook C, et al. Diagnostic accuracy and association to disability of clinical test findings associated with patellofemoral pain syndrome. Physiother Can. 2010;62(1):17-24.

40. Wagemakers HP, et al. Diagnostic accuracy of history taking and physical examination for assessing anterior cruciate ligament lesions of the knee in primary care. Arch Phys Med Rehabil. 2010;91(9):1452-9.

41. Wagemakers HP, et al. Diagnostic value of history-taking and physical examination for assessing meniscal tears of the knee in general practice. Clin J Sport Med. 2008;18(1):24-30.

42. Kastelein $\mathrm{M}$, et al. Diagnostic value of history taking and physical examination to assess effusion of the knee in traumatic knee patients in general practice. Arch Phys Med Rehabil. 2009;90(1):82-6.

43. Kastelein $\mathrm{M}$, et al. Assessing medial collateral ligament knee lesions in general practice. Am J Med. 2008;121(11):982-8. e2

44. MacKay C, et al. Expanding roles in orthopaedic care: a comparison of physiotherapist and orthopaedic surgeon recommendations for triage. $J$ Eval Clin Pract. 2009:15(1):178-83.

45. Anderson MJ, et al. A systematic summary of systematic reviews on the topic of the anterior Cruciate ligament. Orthop J Sports Med. 2016;4(3): 2325967116634074

46. Parker BR, et al. Surgical trends in the treatment of Meniscal tears: analysis of data from the American Board of Orthopaedic Surgery Certification Examination Database. Am J Sports Med. 2016;44(7):1717-23.

47. Stensrud S, Risberg MA, Roos EM. Knee function and knee muscle strength in middle-aged patients with degenerative meniscal tears eligible for arthroscopic partial meniscectomy. Br J Sports Med. 2014:48(9):784-8.

48. Hawker $\mathrm{G}$, et al. Perspectives of Canadian stakeholders on criteria for appropriateness for Total joint Arthroplasty in patients with hip and knee osteoarthritis. Arthritis Rheumatol. 2015;67(7):1806-15.

49. Skou ST, et al. Criteria used when deciding on eligibility for total knee arthroplasty - between thinking and doing. Knee. 2016;23(2):300-5.

50. Deyle GD. Direct access physical therapy and diagnostic responsibility: the risk-to-benefit ratio. J Orthop Sports Phys Ther. 2006;36(9):632-4.

51. Hegedus EJ, Moody J. Clinimetrics corner: the many faces of selection bias. J Man Manip Ther. 2010;18(2):69-73. 